\title{
Médiévales
}

Langues, Textes, Histoire

76 | printemps 2019

Du nouveau en archives

\section{JEAN DE VIGNAY, Le Miroir historial, volume I, tome I (livres I-IV)}

éd. Mattia CaVAGna, Paris, Société des anciens textes français, Abbeville, F. Paillart, 2017, 814 p.

\section{Elisabetta Barale}

\section{OpenEdition}

Journals

Édition électronique

URL : https://journals.openedition.org/medievales/10080

DOI : 10.4000/medievales. 10080

ISSN : 1777-5892

Éditeur

Presses universitaires de Vincennes

\section{Édition imprimée}

Date de publication : 5 septembre 2019

Pagination : 167-169

ISBN : 978-2-37924-028-7

ISSN : 0751-2708

\section{Référence électronique}

Elisabetta Barale, «JEAN DE VIGNAY, Le Miroir historial, volume I, tome I (livres I-IV) 》, Médiévales [En ligne], 76 | printemps 2019, mis en ligne le 05 septembre 2019, consulté le 22 avril 2022. URL : http:// journals.openedition.org/medievales/10080; DOI : https://doi.org/10.4000/medievales.10080

Ce document a été généré automatiquement le 22 avril 2022

Tous droits réservés 


\section{JEAN DE VIGNAY, Le Miroir historial, volume I, tome I (livres I-IV)}

éd. Mattia cavagna, Paris, Société des anciens textes français, Abbeville, F. Paillart, 2017, 814 p.

\section{Elisabetta Barale}

\section{RÉFÉRENCE}

JEAN DE VIGNAY, Le Miroir historial, volume I, tome I (livres I-IV), éd. Mattia CAVAGNA, Paris, Société des anciens textes français, Abbeville, F. Paillart, 2017, 814 p.

1 Ce volume constitue le premier volet de l'édition critique de la traduction française du Speculum historiale de Vincent de Beauvais, réalisée vers 1320-1330 par Jean du Vignay pour répondre à la demande d'un membre de la famille royale de France ; le projet d'édition est destiné à occuper cinq volumes - chacun composé de deux tomes - dont quatre pour le texte et un pour le glossaire. Mattia Cavagna édite ici les livres I-IV, qui contiennent la portion d'histoire de l'humanité correspondant à la période comprise entre la création du monde et le $\mathrm{IV}^{\mathrm{e}}$ siècle av. J.-C. Le récit historique, entremêlé de digressions géographiques et généalogiques, d'extraits d'œuvres d'Aristote et d'un petit fablier ésopique, est précédé de la traduction des vingt premiers chapitres du Libellus apologeticus, dans lequel Vincent de Beauvais explique sa méthode et présente ses sources.

2 Le témoin choisi comme manuscrit de base est le manuscrit Paris, BnF, fr. 316 (J1), terminé en 1333 ; il contient la première version, non révisée, de la traduction par Jean de Vignay. À l'intérieur de la tradition textuelle des quatre premiers livres, comprenant onze manuscrits et deux imprimés anciens, les deux autres témoins retenus pour l'édition sont les exemplaires Leyde, Universiteitsbibliotheek, Voss. Gall. Fol. 3A (A1) et Paris, BnF, fr. 312 (Or1), qui portent tous les deux la trace d'une révision effectuée après 1370 par un autre clerc, probablement à l'incitation du roi Charles V. 
3 L'Introduction, constituée de dix chapitres, synthétise et précise les résultats d'études préliminaires consacrées à la tradition textuelle, aux questions lexicales, aux traits stylistiques et aux réélaborations successives de l'ouvrage. Dans les chapitres 1-4, l'éditeur formule des hypothèses convaincantes quant au travail de révision, qui aurait été réalisé directement sur l'original perdu $\mathrm{O}$, puis transposé dans le manuscrit A1 qui contenait à l'origine la première version et qui fut donc corrigé, le plus souvent par grattage, afin d'accueillir les révisions; Mattia Cavagna utilise donc le sigle A1' pour indiquer ce nouvel état du texte. La version révisée fut encore copiée dans le codex Or1, qui ne présente pourtant pas exactement les mêmes passages corrigés figurant dans A1'; cela amène l'éditeur à supposer un travail de révision en deux temps et aussi à postuler que les scribes chargés de la correction de A1 et de la copie de Or1 travaillèrent de manière indépendante, en revenant parfois à la source latine. Quant au stemma proposé pour cette première portion de traduction du Speculum, il comporte une structure dynamique ( $O$ évoluant en $O^{\prime}$ ) à trois branches ; cette reconstruction des rapports généalogiques entre les trois témoins sert de base à l'établissement du texte : le but étant celui de reconstruire le texte sorti de la plume de Jean de Vignay, les leçons de J1 sont donc corrigées, pour les passages non révisés, lorsque l'accord de A1 et Or1 contre J1 permet de supposer une variante originale.

4 Toujours à propos de la révision, une étude dense et fine de la langue du texte menée dans le chapitre 5 de l'Introduction vise à mettre en relief les aspects linguistiques que le réviseur aurait perçus comme obsolètes ou bien si caractéristiques du style de Jean de Vignay qu'ils nécessitaient une "neutralisation ». Dans ce but, Mattia Cavagna choisit de se focaliser sur certaines particularités morphosyntaxiques telles que les traces de déclinaison, les adjectifs épicènes, les compléments du nom en régime direct et les nombreux régionalismes, néologismes et calques dont la langue du traducteur est particulièrement riche. En outre, l'étude des créations verbales, des hapax et des mots utilisés avec un sens nouveau fait ressortir les questions lexicales les plus intéressantes, approfondies dans les notes au texte. Le regroupement des néologismes d'après le type de formation (reprise de termes latins, francisation, adaptation au moyen de suffixes/ préfixes, calques) et le classement typologique des interventions du réviseur (choix subjectifs, élimination d'un latinisme ou d'un mot vieilli, retour à la source latine pour remplacer un mot courant...) met aussi en évidence les orientations stylistiques du réviseur.

Quant à la traduction elle-même, elle est étudiée dans le chapitre 6. Si l'exemplaire du texte latin sur lequel Jean de Vignay a travaillé reste impossible à identifier en raison de la complexité de la tradition et de l'absence d'édition critique de la source, le travail de l'éditeur permet d'identifier plusieurs passages ayant été traduits de manière fautive en raison de l'état du texte de départ, ou d'une lecture par trop rapide du traducteur ; cela a comme conséquence que le jugement souvent très sévère porté sur le travail du clerc normand peut en partie être mitigé. La diversité des sources dont Vincent de Beauvais s'était servi est également indiquée comme une difficulté que le traducteur a dû surmonter pendant son travail ; en effet, la variété des registres, des styles et du vocabulaire caractérisant le texte latin a certainement constitué un obstacle majeur pour la mise en français. Parmi les nombreux mérites de cette édition critique qui repose sur des principes méthodologiques rigoureux et se caractérise par un grand soin, on signalera justement aussi le travail accompli au niveau de l'identification des textes qui servirent à la compilation du Speculum historiale; en effet, l'éditeur a fourni le 
renvoi à tous les auteurs et aux œuvres mentionnés de manière explicite dans le texte, ce qui permet donc de mieux évaluer la traduction elle-même, mais aussi la compilation latine.

6 Les chapitres 7 et 8 de l'Introduction contiennent respectivement le plan du contenu des sections de l'œuvre éditées ici et la description détaillée des trois témoins utilisés pour l'établissement du texte ainsi que l'étude de leurs rapports de filiation. Quant au chapitre 9 , il présente les principes d'édition, dont il a déjà été question en partie. À ce propos, la politique éditoriale concernant les nombreux noms propres est inspirée de la conservation de la forme figurant dans le manuscrit de base, tout comme celle qui intéresse les rubriques et les tables présentes dans J1.

7 L'édition occupe les pages 111-754. La transcription, toujours précise et soignée, est accompagnée d'un commentaire philologique pertinent, ponctuel et très utile pour éclaircir le texte, ainsi que pour apprécier les différents états de la traduction. L'apparat se compose de quatre étages, figurant tous en bas de page. Dans le premier, on trouvera les notes critiques et historiques. Dans celles-ci, on remarquera tout particulièrement la discussion des passages problématiques et surtout celle des questions lexicales, qui permet de pallier l'absence, dans ce volume, d'un glossaire: celui-ci ne sera contenu, en effet, que dans le cinquième tome prévu par le projet intégral. Il est donc d'autant plus important que les notes signalent les hapax, les sens peu attestés, les locutions, et qu'elles fournissent souvent une traduction en français moderne ; elles permettent d'ores et déjà d'effectuer des études ponctuelles et surtout d'enrichir la connaissance du lexique du moyen français. Le deuxième étage de l'apparat contient les indications à propos des corrections du manuscrit de base, tandis que dans le troisième figurent les variantes de A1, A1' et Or1, grâce auxquelles le lecteur peut, entre autres, reconstruire dans le détail le travail de révision. Le quatrième étage contient les notes à propos de l'identification des sources compilées par Vincent de Beauvais. L'index des noms propres (p. 757-812), qui achève le volume, permet de consulter le texte confortablement.

8 La bibliographie, particulièrement riche, constitue le chapitre 10 de l'Introduction: elle rendra sans aucun doute de grands services à ceux qui s'intéressent à l'encyclopédisme médiéval, à la traduction dans ses nombreuses facettes et plus généralement à la production littéraire en moyen français.

\section{AUTEURS}

\section{ELISABETTA BARALE}

Università degli Studi di Torino 